\title{
A Dialectica-like Model of Linear Logic
}

\author{
Valeria C. V. de Paiva \\ Dept. Informatica PUC-RJ \\ R.M.S.Vicente, 225 - Rio de Janeiro 22453
}

BRAZIL

The aim of this work is to define the categories GC, describe their categorical structure and show they are a model of Linear Logic. The second goal is to relate those categories to the Dialectica categories DC, cf.[DC], using different functors for the exponential "of course". It is hoped that this categorical model of Linear Logic should help us to get a better understanding of the logic, which is, perhaps, the first non-intuitionistic constructive logic.

This work is divided in two parts, each one with 3 sections. The first section shows that GC is a monoidal closed category and describes bifunctors for tensor " $\oslash$ ", internal hom " $[-,-]$ ", par " $\sharp$ ", cartesian products " $\&$ " and coproducts " $\oplus$ ". The second section defines linear negation as a contravariant functor obtained evaluating the internal hom bifunctor at a "dualising object". The third section makes explicit the connections with Linear Logic, while the fourth introduces the comonads used to model the connective "of course". Section 5 discusses some properties of these comonads and finally section 6 makes the logical connections once more.

This work grew out of suggestions of J.Y. Girard at the AMS-Conference on Categories, Logic and Computer Science in Boulder 1987, where I presented my earlier work on the Dialectica categories, hence the title. Still on the lines of given credit where it is due, I would like to say that Martin Hyland, under whose supervision this work was written, has been a continuous source of ideas and inspiration. Many heartfelt thanks to him.

\section{The main deflnitions}

We start with a finitely complete category $\mathbf{C}$. Then to describe $\mathbf{G C}$ say that its objects are relations on objects of $\mathbf{C}$, that is monics $A \stackrel{\alpha}{\rightarrow} U \times X$, which we usually write as $(U \stackrel{\alpha}{+} X)$.

Given two such objects, $(U \stackrel{\alpha}{+} X)$ and $(V \stackrel{\beta}{+} Y)$, which we call simply $A$ and $B$, a morphism from $A$ to $B$ consists of a pair of maps in $\mathbf{C}, f: U \rightarrow V$ and $F ; Y \rightarrow X$, such that a pullback condition is satisfied, namely that

$$
(U \times F)^{-1}(\alpha) \leq(f \times Y)^{-1}(\beta)
$$

where $(-)^{-1}$ represents pullbacks. 
Using diagrams, we say $(f, F)$ is a morphism in GC if there is a (unique) map in $\Sigma, k: A^{\prime} \rightarrow B^{\prime}$ making the triangle commute:

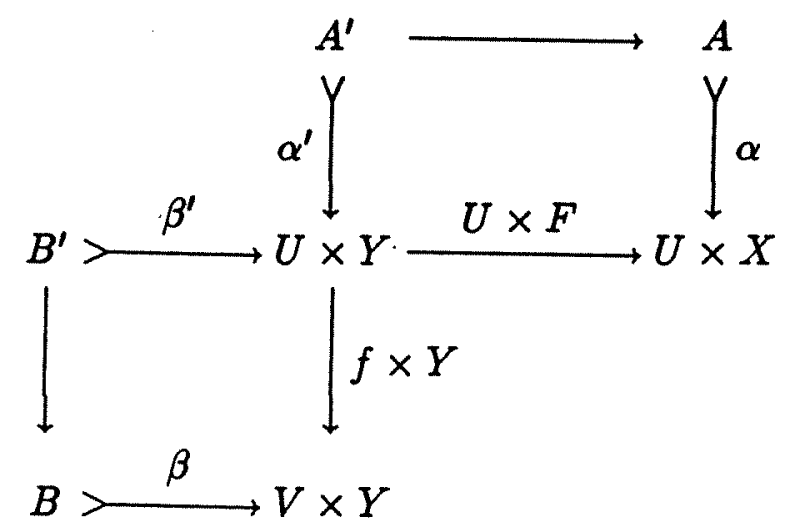

here $A^{\prime}$ is the pullback of $\alpha$ along $U \times F$ and $B^{\prime}$ the pullback of $\beta$ along $f \times Y$. Note lat we refer to the object $(U \stackrel{\alpha}{+} X)$ as " $\alpha$ ", meaning the (equivalence class of the) ionic, as well as $A$.

The intuition here is that, if we consider $\alpha$ and $\beta$ set-theoretic relations, there is a corphism from $\alpha$ to $\beta$

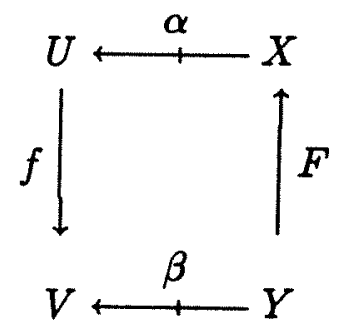

whenever $u \alpha F(y)$ then $f(u) \beta y$.

It is easy to see GC is a category, since composition is just composition in each oordinate'.

If the base category $\mathbf{C}$ is cartesian closed, as well as finitely complete, the category $\mathbf{C}$ has a symmetric monoidal structure (tensor product) denoted by " $\oslash$ " that can be ade closed. This tensor bifunctor $\oslash$ seems somewhat involved and not very intuitive, it it is exactly what is needed to show that GC is symmetric monoidal closed, given e internal hom, $[-,-]_{G C}$ defined below.

eflnition 1. There is an internal hom bifunctor in GC, $[-,-]_{\mathbf{G C}}: \mathbf{G C}^{\mathbf{O p}} \times \mathbf{G C} \rightarrow \mathbf{G C}$ ven by

$$
[A, B]_{G C}=\left(V^{U} \times X^{Y} \stackrel{\beta^{\alpha}}{\leftarrow U} \times Y\right)
$$


where intuitively the relation $\beta^{\alpha}$ reads as $(f, F) \beta^{\alpha}(u, y)$ iff whenever $u \alpha F(y)$ then $f(u) \beta y$.

Formally, we define $\beta^{\alpha}$ as the greatest subobject $E$ of $V^{U} \times X^{Y} \times U \times Y$ such that $E \wedge A^{\prime} \leq B^{\prime}$, where $A^{\prime}$ is the pullback of $A$ along the map

$$
V^{U} \times X^{Y} \times U \times Y \stackrel{\left(\pi_{3}, " e v^{\prime \prime}\right)}{\longrightarrow} U \times X
$$

$B^{\prime}$ is the pullback of $B$ along $V^{U} \times X^{Y} \times U \times Y \stackrel{\left(" e v^{\prime}, \pi_{4}\right)}{\longrightarrow} V \times Y$ and " $\wedge$ " means pullback again.

To guarantee the existence of such greatest subobject, we insist on $\mathbf{C}$ being locally cartesian closèd: By that we mean that for any object $A$ of $\mathrm{C}$, the slice category $\mathrm{C} / A$ is cartesian closed, cf. [See] 1984.

Definition 2. Assuming $\mathbf{C}$ locally cartesian closed, consider the tensor product in GC given by the operation $\oslash: \mathbf{G C} \times \mathbf{G C} \rightarrow \mathbf{G C}$ which takes the pair of objects $(A, B)$ to

$$
A \oslash B=\left(U \times V \stackrel{\alpha \oslash \beta}{+} X^{V} \times Y^{U}\right) .
$$

Intuitively, $(u, v) \alpha \oslash \beta(f, g)$ iff $\quad u \alpha f(v)$ and $v \beta g(u)$.

The functor " $\theta$ " is not a categorical product, for example, projections do not exist necessarily, but it is associative and symmetric. The object $I=(1 \stackrel{z}{+2})$ is the unit for this tensor product.

Another tensor product, similar to the tensor bifunctor in DC can be defined, but it is not left-adjoint to the internal hom.

Definition 3. The bifunctor $\otimes: \mathbf{G C} \rightarrow \mathbf{G C}$, which takes $(A, B)$ to

$$
A \otimes B=(U \times V \stackrel{\alpha \otimes \beta}{\leftrightarrow} X \times Y)
$$

is associative and symmetric. It has the same unit $I=(1+1)$ as the bifunctor " $\oslash$ " and intuitively the relation $(u, v) \alpha \otimes \beta(x, y)$ holds iff $u \alpha x$ and $v \beta y$.

Proposition 1. The category GC is a symmetric monoidal closed category.

Proof: It's enough to see the natural isomorphism

$$
\operatorname{Hom}_{\mathbf{G C}}(A \oslash B, C) \cong \operatorname{Hom}_{\mathbf{G C}}\left(A,[B, C]_{\mathbf{G C}}\right) \text {. }
$$

Using diagrams,
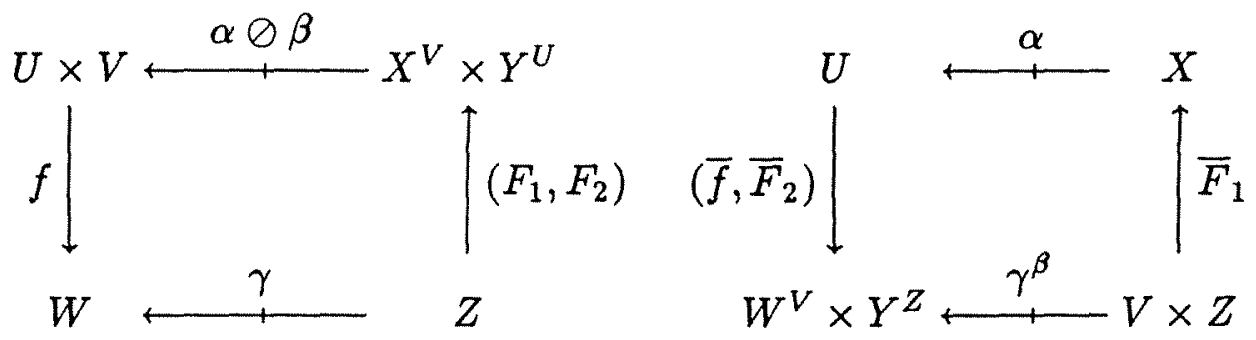
For symmetry reasons that will be apparent later, we want to introduce yet another bifunctor, to be called "\#", which is, in some sense, dual to the tensor product " $\oslash$ " bifunctor. To define the bifunctor "\#" categorically we need $\mathbf{C}$ with stable (under pullbacks) and disjoint coproducts cf. [M/R] 1977.

Definition 4. Consider the bifunctor "\#" that takes $(A, B)$ to

$$
A \sharp B=\left(U^{Y} \times V^{X} \stackrel{\alpha \sharp \beta}{\longleftarrow} X \times Y\right) .
$$

The relation defining $A \sharp B$ says that $(f, g) \alpha \sharp \beta(x, y)$ iff $\quad f(y) \alpha x$ or $g(x) \beta y$.

Notice that the object $\perp=(1 \stackrel{0}{+} 1)$, where 0 is the empty relation on $1 \times 1$, is the unit for the operation " $\sharp$ " and that there is a natural map $\perp \rightarrow I$, but not conversely.

Notice as well that tensor " $\oslash$ " and its dual " $\sharp$ " have very similar "carriers", but duality here is transforming the metalanguage "and" into "or".

Now we define cartesian products and coproducts in GC.

Proposition 2. If $\mathrm{C}$ is a finitely complete category with disjoint and stable coproducts then GC has categorical products and coproducts.

Proof: Categorical products are given by the bifunctor $\&: \mathbf{G C} \times \mathbf{G C} \rightarrow \mathbf{G C}$, which takes the pair of objects $(A, B)$ to

$$
A \& B=(U \times V \stackrel{\alpha \& \beta}{\longleftarrow} X+Y)
$$

and the relation " $\alpha \& \beta$ " is given, intuitively, by $(u, v) \alpha \& \beta\left(\begin{array}{l}x, 0 \\ y, 1\end{array}\right)$ iff either $u \alpha x$ or $v \beta v$. Categorically, we take the coproduct map induced by the morphisms $A \times V \stackrel{\alpha \times V}{\longrightarrow} U \times$ $X \times V$ and $B \times U \stackrel{\beta \times U}{\longrightarrow} V \times Y \times U$. The object $A \& B$ is a cartesian product, as can easily be checked and the object $1=(1 \stackrel{e}{+} 0)$ is the unit for the cartesian product and so a terminal object in GC.

The construction above can be dualised. Thus, if we take the coproduct map of $A \times Y \stackrel{\alpha \times Y}{\longrightarrow} U \times X \times Y$ and $B \times X \stackrel{\beta \times X}{\longrightarrow} V \times Y \times X$ that gives us

$$
A \oplus B=(U+V \stackrel{\alpha \oplus \beta}{\longleftarrow} X \times Y)
$$

where the natural relation reads as $\left(\begin{array}{l}u, 0 \\ v, 1\end{array}\right) \alpha \oplus \beta(x, y)$ iff either $u \alpha x$ or $v \beta y$. Clearly the endofunctor $\oplus$ defines coproducts in GC. The object $\mathbf{0}=(0 \stackrel{e}{+} 1)$ is the unit for this construction.

A remark is that the intuitive "or" in the definitions of $\&$ and $\oplus$ is given by taking coproducts, while the one in the definition of $\sharp$ is a real "or". 


\section{Linear negation in GC}

We define in GC a strong contravariant functor, which induces an involution on a subcategory of GC.

Recall that, given a symmetric monoidal closed category $\mathbf{C}$, a contravariant strong functor $T: \mathbf{C} \rightarrow \mathbf{C}$ is a functor such that, for $\Omega$ every pair of objects $(A, B)$ in $\mathbf{C}$, there is a family of maps $s t_{(A, B)}:[A, B]_{\mathbf{C}} \rightarrow[T B, T A]_{\mathbf{C}}$ making the following diagrams commute.

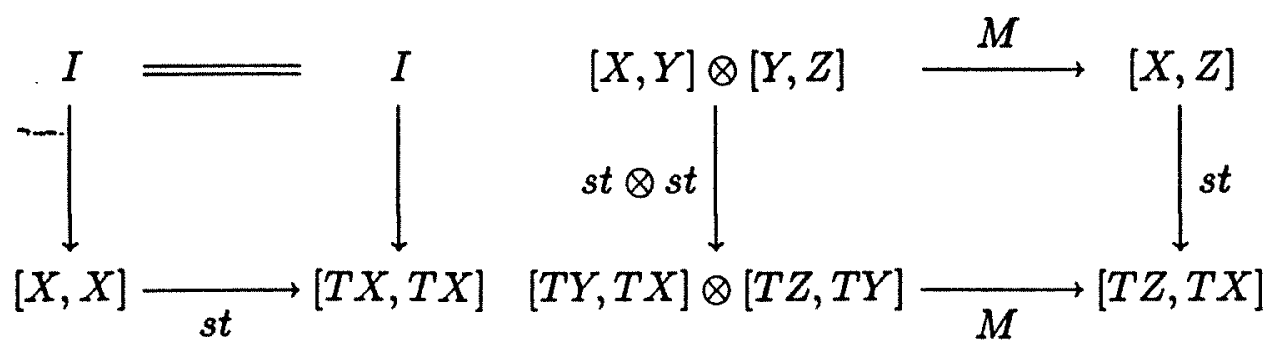

Definition 5. Consider the internal hom bifunctor evaluated at $\perp=\left(\begin{array}{ll}1 \stackrel{0}{\leftarrow} & 1\end{array}\right)$ in the second coordinate, that is consider $[-, \perp]_{\mathbf{G C}}$. This obviously defines a contravariant functor $(-)^{\perp}: \mathbf{G C}^{\text {op }} \rightarrow \mathbf{G C}$.

More precisely to each object $(U \stackrel{\alpha}{+} X)$, the functor $(-)^{\perp}$ associates the object $\left(X \stackrel{1^{\alpha}}{\longleftarrow} U\right)$ where the relation " $\perp^{\alpha}$ " intuitively says $x \perp^{\alpha} u$ iff whenever $u \alpha x$ then $\perp$. As " $\perp$ " is the empty relation, it is never the case, so if we are dealing with decidable relations in Sets, $x \perp^{\alpha} u$ iff it is not the case that $u \alpha x$. Hence the name linear negation.

Proposition 3. The functor $(-)^{\perp}: \mathbf{G C}^{o p} \rightarrow \mathbf{G C}$ is a strong contravariant functor.

Now we want to consider the subcategory "Dec GC", whose objects are the decidable objects in GC, that is decidable relations on $\mathbf{C}$.

Definition 6. By a decidable object on GC we mean that $(U \stackrel{\alpha}{+} X)$ is such that the canonical map from $(U \stackrel{\alpha}{+} X)$ to $\left(U \stackrel{+1}{\leftarrow}^{\alpha} X\right)$ is an isomorphism.

Our next proposition is to give names to structures. Following Barr, cf. [Bar] page 13 , we say that a $*$-autonomous category comprises:

1. A symmetric monoidal closed category $\mathbf{C}$.

2. A strong (contravariant) functor $*: \mathrm{C}^{o p} \rightarrow \mathrm{C}$, thus the functor $*$ and a family of maps $s t^{*}:[A, B]_{\mathbf{G C}} \rightarrow\left[B^{*}, A^{*}\right]_{\mathbf{G C}}$.

3. An equivalence $d=d A: A \rightarrow A^{* *}$ such that the following diagram commutes

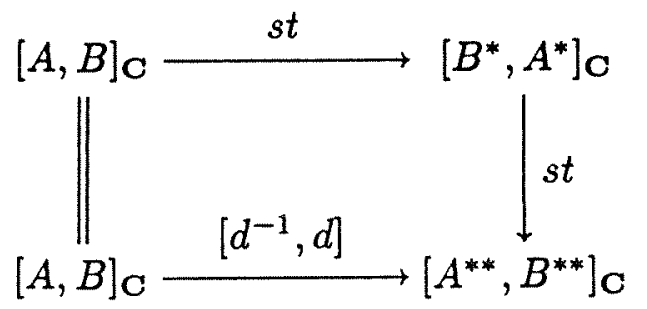


Proposition 4. The subcategory Dec GC is an $*$-autonomous category, for $*=(-)^{\perp}$

It is easy to verify that the tensor product " $\oslash$ " of GC distributes over the coproduc' $\oplus$ and dually, that the bifunctor par "\#" distributes over the cartesian product "\&" The following isomorphisms hold in GC

$$
A \oslash(B \oplus C) \cong(A \oslash B) \oplus(A \oslash C) \text { and } A \sharp(B \& C) \cong(A \sharp B) \&(A \sharp C) \text {. }
$$

Notice that 'multiplicatives' distribute over 'additives'. But there are also natura morphisms of the form $\left(A \oslash A^{\prime}\right) \oslash(B \sharp C) \stackrel{k}{\longrightarrow}(A \oslash B) \sharp\left(A^{\prime} \oslash C\right)$ and symmetrically $(A \sharp B) \oslash\left(C \oslash C^{\prime}\right) \stackrel{k^{\prime}}{\longrightarrow}(A \oslash C) \sharp\left(B \oslash C^{\prime}\right)$, which reduce to the morphisms $A \oslash(B \sharp C) \stackrel{i}{-}$ $(A \oslash B) \sharp C$ and $(A \sharp B) \oslash C^{\prime} \stackrel{i^{\prime}}{\longrightarrow} A \sharp\left(B \oslash C^{\prime}\right)$.

\section{Classical Linear Logic and GC}

The category GC came into existence aiming to be a categorical model of Classica Linear Logic. It stems from a suggestion of Girard in Boulder 87, to whom I am very grateful, and to a great extent it fulfils its promise. In particular, the category GC is : very interesting model of Classical Linear Logic, since it does not collapse the units $o$ : "tensor" and "par" into a single object.

There are at least two equivalent presentations of Classical Linear Logic with slight variations in notation.

The original one, cf. [Gir] 1986 page 22, is very sleek and elegant, but it is hard tc read off a categorical model from it.

Seely in [See] 1987, on the other hand, gives a presentation, which is geared towards the symmetries and thus more helpful. In his presentation a sequent has the form

$$
G_{1}, G_{2}, \ldots, G_{n} \vdash D_{1}, D_{2}, \ldots, D_{m},
$$

where the commas on the left should be thought as some kind of conjunction and those on the right, some kind of disjunction.

A (propositional) Classical Linear Logic consists of formulae and sequents. For mulae are generated by the binary connectives $\otimes, \sharp, \&, \oplus$ and $\multimap$ and by the unary operation $(-)^{\perp}$, from a set of constants including $I, \perp, 1$ and $\mathbf{0}$ and from variables.

The sequents are generated by the following rules, from initial sequents or axioms

Axioms:

$$
\begin{aligned}
& A \vdash A \text { (identity) } \\
& \vdash I \quad \perp \vdash \\
& \Gamma \vdash 1, \Delta \quad \Gamma, \bigcirc \vdash \Delta \\
& A \vdash A^{\perp \perp} \quad A^{\perp \perp \vdash A} \quad \text { (negation) }
\end{aligned}
$$

Structural Rules:

$$
\frac{\Gamma \vdash \Delta}{\sigma \Gamma \vdash \tau \Delta} \text { (permutation) } \quad \frac{\Gamma \vdash A, \Delta \quad A, \Gamma^{\prime} \vdash \Delta^{\prime}}{\Gamma, \Gamma^{\prime} \vdash \Delta^{\prime}, \Delta} \text { (cut) }
$$


Logical Rules:

$$
\frac{\Gamma, A \vdash B, \Delta}{\Gamma, B^{\perp} \vdash A^{\perp}, \Delta} \text { (var) }
$$

Multiplicatives:$$
\left(\text { unit }_{l}\right) \frac{\Gamma \vdash \Delta}{\Gamma, I \vdash \Delta}
$$$$
\left(\text { unit }_{r}\right) \frac{\Gamma \vdash \Delta}{\Gamma \vdash \perp, \Delta}
$$$$
\left(\otimes_{I}\right) \frac{\Gamma, A, B \vdash \Delta}{\Gamma, A \otimes B \vdash \Delta}
$$$$
\left(\otimes_{r}\right) \frac{\Gamma \vdash A, \Delta \quad \Gamma^{\prime} \vdash B, \Delta^{\prime}}{\Gamma, \Gamma^{\prime} \vdash A \otimes B, \Delta, \Delta^{\prime}}
$$$$
(\sharp) \frac{\Gamma, A \vdash \Delta \quad \Gamma^{\prime}, B \vdash \Delta^{\prime}}{\Gamma, \Gamma^{\prime}, A \sharp B \vdash \Delta, \Delta^{\prime}}
$$$$
\left(\sharp_{r}\right) \frac{\Gamma \vdash A, B, \Delta}{\Gamma \vdash A \sharp B, \Delta}
$$$$
(\multimap,) \frac{\Gamma \vdash A, \Delta \quad \Gamma^{\prime}, B \vdash \Delta^{\prime}}{\Gamma, \Gamma^{\prime}, A \multimap B \vdash \Delta^{\prime}, \Delta}
$$$$
(\multimap r) \frac{\Gamma, A \vdash B, \not}{\Gamma \vdash A \multimap B, \not}
$$

Additives:

$$
\begin{array}{ccc}
\left(\&_{r}\right) \frac{\Gamma \vdash A, \Delta \quad \Gamma \vdash B, \Delta}{\Gamma \vdash A \& B, \Delta} & \left(\&_{l}\right) \frac{\Gamma, A \vdash \Delta}{\Gamma, A \& B \vdash \Delta} & \frac{\Gamma, B \vdash \Delta}{\Gamma, A \& B \vdash \Delta} \\
\left(\oplus_{l}\right) \frac{\Gamma, A \vdash \Delta \quad \Gamma, B \vdash \Delta}{\Gamma, A \oplus B \vdash \Delta} & \left(\oplus_{r}\right) \frac{\Gamma \vdash A, \Delta}{\Gamma \vdash A \oplus B, \Delta} & \frac{\Gamma \vdash B, \Delta}{\Gamma \vdash A \oplus B, \Delta}
\end{array}
$$

A remark on notation. Seely writes in his paper " $x$ " for " $\&$ ", " + " for " $\oplus$ ", $\odot$ for $\sharp$ and $\neg$ for $(-)^{\perp}$, but we want to keep, as much as possible, the original notation from [Gir].

We would like GC with all the structure defined before, to be a categorical model of Classical Linear Logic. But it is clear that we do not have morphisms of the form $A^{\perp \perp} \rightarrow A$ for all objects $A$ in GC. So, not all the objects are equivalent to their double linear negations, $A \cong A^{\perp \perp}$.

Thus, we omit from the system just presented the negation axiom $A^{\perp \perp} \vdash A$. It is interesting to note that, apart from the axiom, we only have to change the negation rule (var). Actually we transform the rule (var) into two rules, the rules (varl) and (var $)$ as below,

$$
\left(v_{r}\right) \frac{\Gamma, A \vdash \Delta}{\Gamma \vdash A^{\perp}, \Delta} \quad\left(v_{i}\right) \frac{\Gamma \vdash B, \Delta}{\Gamma, B^{\perp} \vdash \Delta}
$$


Then only the rule (varl) is satisfied in GC. That happens because the lo are dealing with is really intuitionistic, at the bottom level. Thus, for example, model, the objects $(A \oslash B)^{\perp}$ and $\left(A^{\perp} \sharp B^{\perp}\right)$ "look" exactly the same; they are b the form $\left(X^{V} \times Y^{U} \leftrightarrow U \times V\right)$. But taking in consideration the relations, $w$ have a morphism in one direction: $\left(A^{\perp} \sharp B^{\perp}\right) \vdash(A \oslash B)^{\perp}$. This is just as it case in Intuitionistic Logic, thinking of "\#" as "or" and " $\oslash$ " as "and". Look the rule $\left(v a r_{r}\right)$ we see that it is not satisfied in general, since if we have a mor $G \oslash A \rightarrow D$, that is equivalent to a morphism $G \rightarrow(A \multimap D)$. But from $A^{\perp} \sharp D$ 、 prove intuitionistically, $A \multimap D$, but not conversely. Thus we have to leave out (1

Let the new logical system, without $A^{\perp \perp} \vdash A$ and $\left(\right.$ var $\left._{r}\right)$, be called $L . L_{\star}$ or times just L.L.

Theorem. The symmetric monoidal closed category GC, with bifunctors tensor uct $\oslash$; "par" \#; internal hom [-,-] $]_{\mathbf{G C}}$; cartesian product \&; coproduct $\oplus$ anı travariant functor $(-)^{\perp}$ for linear negation, is a model of $L . L_{\star}$. Thus to each ment $\Gamma \vdash_{L . L_{*}} A$ corresponds the existence of a morphism in GC, $(f, F):|\Gamma| \rightarrow$ $|\Gamma| \vdash_{\mathbf{G C}}|A|$.

The proof is merely to check each of the axioms and rules.

Notice that rules $\oslash_{l}$ and $\sharp_{r}$ are fundamental, since they indicate how we s interpret the sequents in the category GC. They show that

$$
G_{1}, G_{2}, \ldots, G_{n} \vdash D_{1}, D_{2}, \ldots, D_{m}
$$

should be read as there exists a morphism in GC,

$$
\left|G_{n}\right| \oslash \ldots \oslash\left|G_{1}\right| \rightarrow\left|D_{k}\right| \sharp \ldots \sharp\left|D_{1}\right|
$$

Corollary. The subcategory "Dec GC" is a model of Classical Linear Logic. 
).

re-

d -

in-

iil-

or

Id

\section{Modalities in GC}

Interpretations of the modal, or exponential, operators "!" and "?" of Linear Logic, in a categorical set-up, should correspond to a comonad and a monad, respectively, satisfying certain conditions. We discuss endofunctors on $\mathbf{G C}$, which could play the role of the connective "!" in Classical Linear Logic. The first idea was, following the model of DC, to look at free monoids in $\mathbf{C}$ and see whether they would induce appropriate comonoids in GC.

\section{Some definitions}

Consider the monad given by the construction of free-monoids in $\mathrm{C}$. Thus, suppose we are given an adjunction $F \dashv U: \mathbf{C} \rightarrow$ Mon $\mathbf{C}$ and call $S_{0}$, or alternatively *, the composition $F U: \mathbf{C} \rightarrow \mathbf{C}$.

Intuitively $X^{*}$ stands for "finite sequences of elements of $X$ " and $f^{*}$ for " $f$ applied to each element of the sequence". Also $S_{0}$ has clearly a monad structure and it does not preserve products. Despite that $S_{0}$ induces an endofunctor $S: \mathbf{G C} \rightarrow \mathbf{G C}$ which has a natural structure as a comonad.

Definition 1. The endofunctor $S$ is given by $S(U \stackrel{\alpha}{\leftrightarrow} X)=\left(U \stackrel{S \alpha}{\leftrightarrow} X^{*}\right)$ on objects. The relation " $S \alpha$ " is given by the pullback below

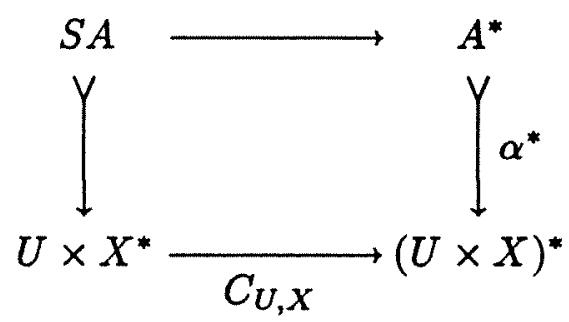

where the auxiliary maps $C_{(-,-)}$are given by the serie of equivalences:

$$
\begin{gathered}
\frac{V \times Y \stackrel{\eta_{(V \times Y)}}{\longrightarrow}(V \times Y)^{*}}{Y \stackrel{\bar{\eta}}{\longrightarrow}(V \times Y)^{* V}} \\
\frac{Y^{*} \stackrel{\bar{C}}{\longrightarrow}(V \times Y)^{* V}}{V \times Y^{*} \stackrel{C_{(V, Y)}}{\longrightarrow}(V \times Y)^{*}}
\end{gathered}
$$

The relation " $S \alpha$ " reads intuitively as

$$
\text { " } u(S \alpha)\left(x_{1}, \ldots, x_{k}\right) \text { iff } u \alpha x_{1} \text { and } \ldots \text { and } u \alpha x_{k} "
$$

and $S$ applied to a morphism $(f, F)$ in $\mathbf{G C}$ is $\left(f, F^{*}\right)$.

The functor $S: \mathbf{G C} \rightarrow \mathbf{G C}$ has a natural comonad structure, induced by the monad structure of $S_{0}$. Alas, this comonad has not the nice categorical properties it had with respect to the categories $\mathbf{D C}$, due to the fact that the tensor product in $\mathbf{G C}$ is much more complicated than the one in DC. There are other very natural monads to consider in $\mathbf{C}$, if $\mathbf{C}$ is cartesian closed. 
Definition 2. For each $U$ in $\mathbf{C}$, let $T_{U}: \mathbf{C} \rightarrow \mathbf{C}$ be the endofunctor which takes $X^{U}, Y \mapsto Y^{U}$ and $f \in X^{U}$ to $f g \in Y^{U}$.

That is clearly a monad in $C$ with unit $\left(\eta_{T_{U}}\right)_{X}: X \rightarrow X^{U}$ given by the "cor map", and multiplication $\left(\mu_{T_{U}}\right)_{X}: X^{U \times U} \rightarrow X^{U}$, simply "precomposition with c nal". We now turn our attention to defining a comonad in GC "induced by the $\mathrm{m}$ " $T_{U}$ ".

Definition 3. Consider the functor $T: \mathbf{G C} \rightarrow \mathbf{G C}$ which takes $(U \stackrel{\alpha}{+} X)$ to the $\left(U \stackrel{T \alpha}{+} X^{U}\right)$ and the object $(V \stackrel{\beta}{+} Y)$ to $\left(V \stackrel{T \beta}{\leftrightarrow} Y^{V}\right)$. The relation "T $\alpha$ " is defin the pullback below

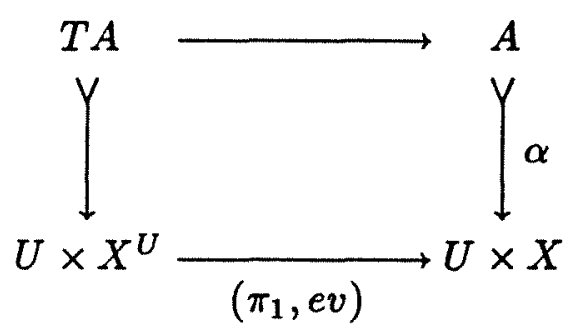

Intuitively, it says that " $u(T \alpha) g$ iff $u \alpha g(u)$ ", where $g \in X^{U}$. To complete the defir say that $T$ applied to a map $(f, F): A \rightarrow B$ is $(f, F(-) f): T A \rightarrow T B$.

It is easy to show that $T$ has a comonad structure induced by the monad struc of the functors $T_{U}$.

Moreover, the monads $T_{U}$ relate to $S_{0}$ in a very special way, described by [69] as a "distributive law". More interesting is the fact that $\lambda$ above, induces : distributivity law $\Lambda$, this time between the comonads $T$ and $S$ in GC.

Proposition 1. For each $U$ and $X$ in $\mathbf{C}$, we have a natural transformation

$$
\lambda_{X}: S_{0} T_{U} X \rightarrow T_{U} S_{0} X
$$

corresponding to $\left(X^{U}\right)^{*} \rightarrow\left(X^{*}\right)^{U}$, satisfying the appropriate diagrams for a distrit law. There is also a natural transformation $\Lambda: T S \rightarrow S T$, at each object $A, \Lambda_{A}: T$ s $S T A$ is given by $\left(1_{U},(\lambda)_{X}\right)$ where $(\lambda)_{X}:\left(X^{U}\right)^{*} \rightarrow\left(X^{*}\right)^{U}$ is the distributive $I$. C. This natural transformation $\Lambda$ satisfies the conditions for a "distributive 1 . comonads".

\section{Using Distributive Laws}

It is widely known that the composition of monads is not always a monad given a distributive law $\lambda$, we can define the composite monad defined by $\lambda$, cf. [F We can also define the "lifting" of one of the monads and several relationships a the categories of algebras and Kleisli categories involved. 
Definition 4. The composite monad $\left(T_{U} S_{0}\right)_{\lambda}$ in $\mathbf{C}$, takes $X \mapsto\left(X^{*}\right)^{U}$. Similarly, we have the composite comonad, induced by $\Lambda$ and given by $(T S): \mathbf{G C} \rightarrow \mathbf{G C}$, which takes

nt

(2)

ds

ct

by

on

es

ck

3W

ve

$\rightarrow$

in

of

ut

t].

$\mathbf{1 g}$ $(U \stackrel{\alpha}{+} X)$ to $\left(U \stackrel{T S \alpha}{\leftrightarrow}\left(X^{*}\right)^{U}\right)$.

Besides the "composite monad", a distributive law provides a "lifting" of one of the monads to the category of algebras for the other monad.

Proposition 2. The monad $T_{U}: \mathrm{C} \rightarrow \mathrm{C}$ lifts to the category of $S_{0}$-algebras. Dually, the comonad $T$ in GC lifts to the category of $S$-coalgebras.

We-can describe another monad $\widetilde{T_{U}}: \mathbf{C}^{\mathbf{S}_{\mathbf{o}}} \rightarrow \mathbf{C}^{\mathbf{S}_{\mathbf{o}}}$. The endofunctor $\widetilde{T_{U}}$ applied to an $S_{0}$-algebra $\left(X, j: X^{*} \rightarrow X\right)$, gives ths $S_{0}$-algebra $\left(X^{U}, h\right)$. The new structural map $h:\left(X^{U}\right)^{*} \rightarrow X^{U}$ is given by the composition $\left(X^{U}\right)^{*} \stackrel{\lambda}{\rightarrow}\left(X^{*}\right)^{U}{\stackrel{\left(j_{X}\right)}{\rightarrow}}^{U} X^{U}$. The endofunctor $\widetilde{T}:(\mathbf{G C})^{S} \rightarrow(\mathbf{G C})^{S}$ has a comonad structure given by the monad structure of $\tilde{T}_{U}$.

Clearly composing the functor $T_{U}$ with the natural transformation $\eta: I \rightarrow S_{0}$ we have a monad morphism $\alpha: T_{U} \rightarrow T_{U} S_{0}$ which takes $X^{U} \mapsto\left(X^{*}\right)^{U}$. We also write $\beta$ for $S_{0} \rightarrow T_{U} S_{0}$, which is $\left(\eta_{T_{U}}\right)$ applied to the functor $S_{0}$, thus taking $X^{*} \mapsto\left(X^{*}\right)^{U}$.

Similarly, there are comonad morphisms $\delta: T S \rightarrow T$ and $\kappa: T S \rightarrow S$, where for each $A$ in GC, $\delta_{A}: T S A \rightarrow T A$ is given by $\delta_{A}=\left(1_{U}, \alpha_{X}\right)$ and $\kappa_{A}: T S A \rightarrow S A$ by $\kappa_{A}=\left(1_{U}, \beta_{X}\right)$.

Proposition 3. The monad and comonad morphisms above induce: $\mathbf{G C}^{S T}$.

. maps in the categories of algebras, $\bar{\alpha}: \mathbf{C}^{T_{U} S_{0}} \rightarrow \mathbf{C}^{T_{U}}$ and $\bar{\beta}: \mathbf{C}^{T_{U} S_{0}} \rightarrow \mathbf{C}^{S_{0}}$;

. maps in the categories of coalgebras $\bar{\delta}: \mathbf{G C}^{T} \rightarrow \mathbf{G C}^{T S}$ and $\bar{\kappa}: \mathbf{G} \mathbf{C}^{S} \rightarrow \mathbf{G} \mathbf{C}^{T S}$.

Proposition 4. There is an equivalence of categories of algebras,

$$
\Phi_{0}:\left(\mathbf{C}^{S_{0}}\right)^{\widetilde{T}_{U}} \rightarrow \mathbf{C}^{S_{0} T_{U}}
$$

and respectively, of categories of coalgebras $\Phi:\left(\mathbf{G C}^{S}\right)^{\widetilde{T}} \rightarrow \mathbf{G C}^{S T}$.

The proofs of Propositions 2, 3 and 4 given in Beck's paper for algebras translate exactly to the coalgebras case, thus we omit them.

Clearly the monad $S_{0}$ does not lift to the category of $T_{U}$-algebras, since we cannot define the $T_{U}$-structural map for $S_{0} X$ using $\lambda$, but it seems to lift to the $T_{U}$-Kleisli category, $\mathbf{C}_{T_{U}}$. Clearly we are talking about duality once more, but that is a more subtle case. 
Proposition 5. The monad $S_{0}$ "lifts" to the Kleisli category $C_{T_{U}}$. Dually, the comonad $S$ lifts to the Kleisli category $\mathbf{G C}_{\mathbf{T}}$.

The endofunctor $\widetilde{S}_{0}: C_{T_{U}} \rightarrow C_{T_{U}}$ takes $X$ to $X^{*}$ and a morphism in $\mathbf{C}_{T_{U}}, X \rightarrow Y$ corresponding to a map $f: X \rightarrow T_{U} Y$ in $\mathbf{C}$ - to the composition $S_{0}(X) \stackrel{S_{0}\left(f_{0}\right)}{\longrightarrow} S_{0} T_{U}(Y) \stackrel{\lambda}{\rightarrow}$ $T_{U} S_{0}(Y)$ which corresponds to $\widetilde{S_{0}}(f): S_{0} X \rightarrow S_{0} Y$ in $\mathbf{C}_{\mathbf{T}_{\mathrm{U}}}$. The functor $\widetilde{S}_{0}$ has a natural comonad structure.

This is a general consequence of the existence of the distributive law. The point here is that all the propositions above could be read off from Street's paper "The formal theory of monads", by a clever 2-categorically minded reader. But we will not go into the 2-categorical aspects of the theory here.

Using the propositions above we can sum up the results of this section in the four "squares" below. Each square has three sides consisting of adjoint-pairs and the last side given by a natural morphism. In $\mathbf{C}$, relating algebras and Kleisli categories and in GC relating coalgebras and Kleisli categories.
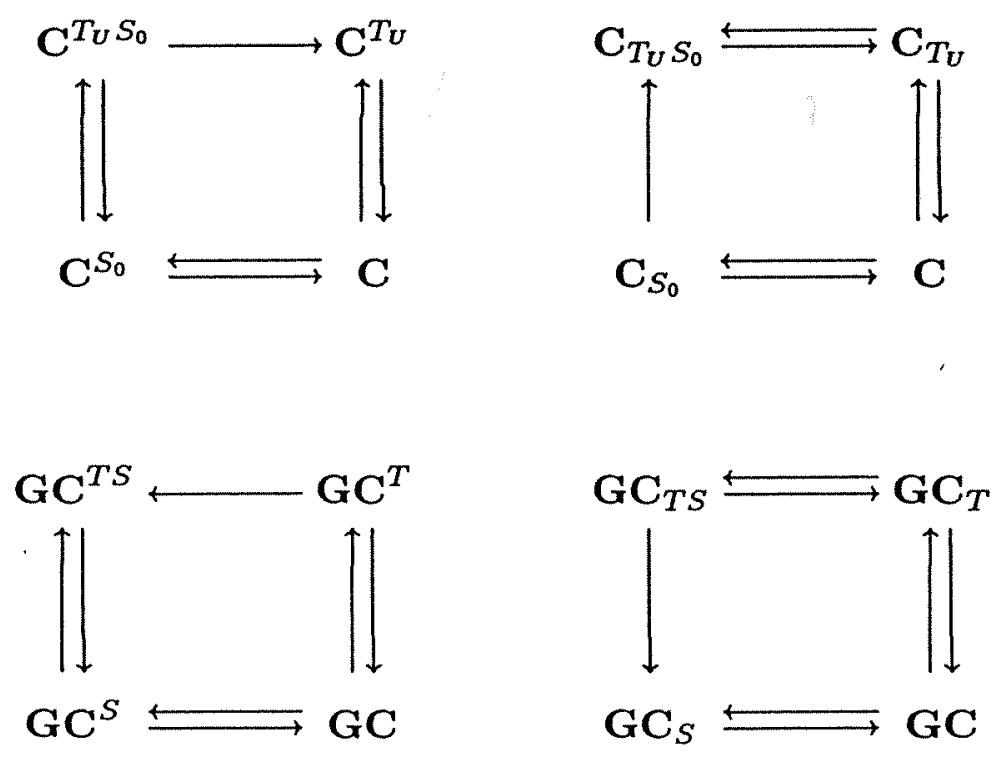

Note that if $\mathbf{C}$ has equalisers then, the two top squares are totally composed of adjoint-pairs, but we do not pursue it here, since it is not clear that equalisers in $\mathbf{C}$ would imply equalisers in GC. 


\section{Properties of the comonads $T$ and !}

In the last section the endofunctor $T: \mathbf{G C} \rightarrow \mathbf{G C}$ was defined and shown to have a natural comonad structure. This endofunctor seems a reasonable candidate to represent the connective "!". For a start it has a "dual" endofunctor, to be denoted $R$, described in the next paragraph.

Definition 5. The endofunctor $R$ takes the object $(U \stackrel{\alpha}{+} X)$ to $\left(U^{X} \stackrel{R \alpha}{+} X\right)$, the object $(V \stackrel{\beta}{+} \underset{\sim}{Y})$ to $\left(V^{Y} \stackrel{R \beta}{\leftrightarrow} Y\right)$ and the map $(f, F): A \rightarrow B$ to the map $(f(-) F, F): R A \rightarrow R B$. The relation " $R \alpha$ " is defined using the pullback of $A \stackrel{\alpha}{\longrightarrow} U \times X$ along the evaluation morphism $U^{X} \times X \stackrel{\left(e v, \pi_{1}\right)}{\rightarrow} U \times X$ and intuitively it says " $g(R \alpha) x$ iff $g(x) \alpha x$ ".

The functor $R$ is the functor part of a monad, with unit $\eta_{A}: A \rightarrow R A$ given by the constant map $\eta_{U}: U \rightarrow U^{X}$ in the first coordinate and identity on $X$. Multiplication $\mu: R^{2} A \rightarrow R A$ is given by "restriction to the diagonal" $\mu_{U}: U^{X \times X} \rightarrow U^{X}$ in the first coordinate and identity on $X$.

We would like to have in GC results for " $T$ " analogous to the ones for "!" in DC. For example, we would like the isomorphism relating categorical products to tensor products $(A \& B) \cong ! A \otimes ! B$. But there is no obvious relationship between $T(A \& B)=$ $\left(U \times V+(X+Y)^{U \times V}\right)$ and $T(A) \oslash T(B)=\left(U \times V+X^{U \times V} \times Y^{U \times V}\right)$. What we do have is a relation between the tensor products in GC.

Proposition 6. There is a natural isomorphism in GC, $T(A \otimes B) \cong T A \oslash T B$.

For a far more interesting result, $\Omega$ recall that the $T$-Kleisli category $\mathbf{G C}_{T}$ has as objects the objects of $\mathbf{G C}$ but as maps from $A$ to $B$, maps in $\mathbf{G C}$ from $T A$ to $B$.

Proposition 7. The maps from $A$ to $B$ in the T-Kleisli category $\mathbf{G C}_{T}$, are in 1-1 correspondence with maps from $A$ to $B$ in the category DC.

Proof: We want to check

$$
\operatorname{Hom}_{\mathbf{G C}_{\mathbf{T}}}(A, B)=\operatorname{Hom}_{\mathbf{G C}}(T A, B) \approx \operatorname{Hom} \mathbf{D C}(A, B) .
$$

The second equivalence holds, since a map $(f, F): T A \rightarrow B$ in GC, corresponds to $f: U \rightarrow V$ and $F: Y \rightarrow X^{U}$, satisfying the condition

$$
(U \times F)^{-1}(\alpha) \leq(f \times Y)^{-1}(\beta) .
$$

That corresponds to $f: U \rightarrow V$ and, by exponential transpose, to $\bar{F}: U \times Y \rightarrow X$, satisfying a corresponding condition (*) that is a map $(f, \bar{F}): A \rightarrow B$ in DC.

Since objects are the same in both categories GC and DC, Proposition 7 implies that there is an equivalence between categories $\mathbf{G C}_{T}$ and $\mathbf{D C}$.

\section{The comonad "!"}

Consider now the composite comonad TS defined in the last section, with the difference that now $S_{0}$ denotes free commutative monoids in C. Thus $S(U \stackrel{\alpha}{\leftrightarrow} X)=$ $\left(U \stackrel{S \alpha}{\leftrightarrow} X^{*}\right)$ and $S$ takes a morphism $(f, F): A \rightarrow B$ to $\left(f, F^{*}\right): S A \rightarrow S B$. Let the 
comonad TS be called "!". The functor part of "!" acts on objects as $(U \stackrel{\alpha}{+} X)=$ $\left(U \stackrel{! \alpha}{\leftrightarrow}\left(X^{*}\right)^{U}\right)$ and on maps $!(f, F)=\left(f, F^{*}(-) f\right)$.

As we have shown "!" is the functor part of the composite comonad

$$
\left(!, \varepsilon !: ! A \rightarrow A, \delta_{!}: ! A \rightarrow ! ! A\right)
$$

and we can consider the categories $\mathbf{G C}$ ! of !-coalgebras and $\mathbf{G C}_{\text {! }}$, the !-Kleisli category Moreover, the objects " $A$ " have a natural comonoid-like structure, with respect to " $\oslash$ "

Proposition 8. There are natural morphisms in GC as follows

1. From the object $! A$ to $I$, given by the terminal map on $U$ and the natural $m a_{i}$ $1 \rightarrow\left(X^{*}\right)^{U}$.

2. From $! A$ to $! A \oslash ! A$, which is given by the diagonal map in $\mathbf{C}, \Delta: U \rightarrow U \times U$ an the map $\theta:\left(X^{*}\right)^{U \times U} \times\left(X^{*}\right)^{U \times U} \rightarrow\left(X^{*}\right)^{U}$.

The map $\theta$ is given, intuitively, by taking a pair of functions $(\phi, \sigma)$, each of then of the form $U \times U \rightarrow X^{*}$, to the product map $\phi \times \sigma$ precomposing it with the diagona in $U$ and post-composing it with the multiplication on $X^{*}$, as follows,

$$
U \stackrel{\Delta}{\longrightarrow} U \times U \stackrel{\langle\phi, \sigma>}{\longrightarrow} X^{*} \times X^{*} \stackrel{\mu_{\bullet}}{\longrightarrow} X^{*} .
$$

Proposition 9. We have the following natural isomorphisms for each $A$ and $B$ in GC $!(A \& B) \cong ! A \oslash ! B$.

Proof: Look at the following series of equivalences :

$$
!(A \& B)=T S(A \& B) \cong T(S A \otimes S B) \cong T S A \oslash T S B=! A \oslash ! B .
$$

Proposition 10. The Kleisli category GC $_{!}$is cartesian closed.

That is an easy corollary of the above, since

$$
\begin{gathered}
\operatorname{Hom}_{\mathbf{G C}:}(A \oslash B, C) \cong \operatorname{Hom}_{\mathbf{G C}}(T S(A \oslash B), C) \cong \operatorname{Hom}_{\mathbf{G C}}(! A \oslash ! B, C) \cong \\
\cong \operatorname{Hom}_{\mathbf{G C}}\left(! A,[! B, C]_{\mathbf{G C}}\right) \cong \operatorname{Hom}_{\mathbf{G C}}\left(A,[B, C]_{\mathbf{G C} !}\right) .
\end{gathered}
$$

Corollary. The morphisms from $A$ to $B$ in the category GC $_{\text {, }}$, correspond naturally $t$. morphisms in the category $\mathrm{DC}_{\mathrm{S}}$ from $A$ to $B$. 


\section{Linear Logic with modalities}

The composite comonad "!" defined in the last section satisfies the rules for the modality "!", but we would like also a monad "?" satisfying the rules for the dual connective, called by Girard "why not ?".

We recall the rules for the modality "!". These are:

$$
\begin{array}{ll}
\text { I. } \frac{\Gamma, \mathrm{A} \vdash \mathrm{B}}{\Gamma, ! \mathrm{A} \vdash \mathrm{B}} \quad \text { (dereliction) } & \text { II. } \frac{\Gamma \vdash \mathrm{B}}{\Gamma, ! \mathrm{A} \vdash \mathrm{B}} \quad \text { (weakening) } \\
\text { III. } \frac{\Gamma, \mathrm{A}, ! \mathrm{A} \vdash \mathrm{B}}{\Gamma, ! \mathrm{A} \vdash \mathrm{B}} \quad \text { (contraction) } & \text { IV. } \frac{! \Gamma \vdash \mathrm{A}}{\Gamma \vdash ! \mathrm{A}} \quad \text { !) }
\end{array}
$$

Theorem. The category GC with the composite comonad "?" defined in Section 5 is a model for Linear Logic enriched with modality "?".

The proof is again to check the rules and it is straightforward. Moreover, using $R$ the dual endofunctor to $T$ we can get a monad to model ?. We just have to compose $R$ with the monad in GC induced by the monad $U \mapsto U^{*}$ in C. The composite monad satisfies all necessary conditions.

\section{Concluding remarks}

To conclude it is perhaps worth mentioning some of the several questions that the work on the categories DC and GC prompts, apart from the ones already mentioned in the introduction.

1. Is there an interesting connection between the categorical models $\mathbf{D C}$ and $\mathbf{G C}$ and Girard's new work on the Geometry of Interactions?

2. Since we think of maps in DC and GC as "linear morphisms", in opposition to the more usual morphisms in the Kleisli categories, can we characterize bilinear maps in this context? There is some interesting work of Kock, but the obvious approach does not work, due to the fact that the comonads "!", or rather, their functor parts, are not strong functors.

3. We have shunned away from the 2-categorical aspects of everything discussed previously, but that is not, probably, the best policy, as was indicated by the need of distributive laws. More to the point, there is a very interesting question of using "spans" instead of relations in the construction of DC and GC, which was suggested by Aurelio Carboni.

4. We have worked only with commutative versions of the connectives, that is with symmetric tensor products, "par" bifunctors etc. There is a interesting case to look at, if this commutativity condition is dropped. On those lines there seems to be some connection with Joyal and Street's work on braided monoidal categories. In particular there is also a preprint by D. N. Yetter on "Quantales and (Noncommutative) Linear Logic".

5. Finally, there is the very promising, but as yet very vague idea of connecting Linear Logic with Concurrency and Parallelism. The idea being that Linear Logic may provide an integrated logic, where one would hope to model computational processes 
in a less ad hoc fashion than it has been up to now. In particular, Petri Nets have been proposed as a model for Linear Logic, cf. [Gir] 1987.

\section{References}

$\begin{array}{ll}\text { [Bar] } 1979 & \begin{array}{l}\text { M. BARR } \\ \text { *-Autonomous Categories, LNM 752, Springer-Verlag, } 1979 .\end{array}\end{array}$

[Bec] 1969 J. BECK

Distributive Laws, in Seminar on Triples and Categorical Homology Theory, LNM 80, Springer-Verlag, 119-140.

[Fox] 1976 T. FOX

Coalgebras and Cartesian Categories, Comm. Alg. (7) 4, 665-667.

[Gir]

J-Y. GIRARD

1986 Linear Logic, Theoretical Computer Science 46, 1-102.

1987 Towards a Geometry of Interactions, to appear in Proc. A.M.S

Conference on Categories in Computer Science and Logic, Boulder, 1987.

[G/L] 1986 J-Y. GIRARD and Y. LAFONT

Linear Logic and Lazy Computation, Proc. of TAPSOFT'87, Pisa.

[Göd] K. GÖDEL

1958 Über eine bisher noch nicht benützte Erweiterung des finiten Standpunktes, Dialectica 12, 280-287.

1980 On a Hitherto Unexploited Extension of the Finitary Standpoint, Journal of Philosophical Logic 9, 133-142.

[Koc] A. KOCK

1970 Monads on Symmetric Monoidal Closed Categories, Arch. Math (21).

1971 Bilinearity and Cartesian Closed Monads, Math. Scand. (29).

[M/R] 1977 M. MAKKAI and G. REYES

First-order Categorical Logic, LNM 611, Springer-Verlag.

[DC] $\quad 1987$ V. C. V. de PAIVA

The Dialectica Categories, to appear in Proc. A.M.S Conference on Categories in Computer Science and Logic, Boulder, 1987.

[See] R. A. G. SEELY

1984 Locally Cartesian Closed Categories and Type Theory, Math. Proc.

Cambridge Philosophical Society 95, 33-48.

1987 Linear Logic, *-autonomous categories and cofree coalgebras to appear in Proc. A.M.S Conference on Categories in Computer Science and Logic, 1987.

[Str] 1972 R. STREET

The formal theory of monads, Journ. of Pure \& Applied Alg. 2, 149-168. 